\title{
Escolares que Convivem com a Depressão Materna Recorrente: Diferenças entre os Sexos
}

\author{
Lívia Loosli \\ Fernanda Aguiar Pizeta \\ Sonia Regina Loureiro ${ }^{1}$ \\ Universidade de São Paulo
}

\begin{abstract}
RESUMO - Há divergências na literatura quanto às diferenças entre os sexos na associação entre depressão materna e desenvolvimento infantil. Neste estudo, compararam-se diferenças entre os sexos relativas ao comportamento e desempenho escolar de crianças que conviviam com a depressão materna, correlacionando tais desfechos. Participaram 40 díades mãecriança, tendo as mães diagnóstico de Transtorno Depressivo Recorrente. As crianças, de sete a 12 anos, foram divididas em grupos por sexo. Foi realizada aplicação de entrevista diagnóstica, do Questionário de Capacidades e Dificuldades e do Teste de Desempenho Escolar. As meninas apresentaram mais sintomas emocionais comparadas aos meninos. Constataram-se associações significativas entre comportamento e desempenho escolar apenas para as meninas, sinalizando maior vulnerabilidade destas meninas.
\end{abstract}

Palavras-chave: depressão, diferenças sexuais (humano), psicologia da criança, relações mãe-criança, rendimento escolar

\section{School-age Children who Live with Recurrent Maternal Depression: Differences between the Sexes}

\begin{abstract}
Research shows divergent data on the differences between the sexes in the association between maternal depression and children development. The objective of this study was to compare differences between the sexes concerning the behavior and school performance of children living with maternal depression, correlating such outcomes. Participants were 40 mother-child dyads, with mothers diagnosed with Recurrent Depressive Disorder. The children, 7 to 12 years old, were divided into groups by sex. The procedures involved the application of diagnostic interview, the Strengths and Difficulties Questionnaire and the School Performance Test. Girls showed more emotional symptoms than boys. Significant associations between behavior and school performance were found for girls only, indicating greater vulnerability of them.
\end{abstract}

Keywords: depression, human sex differences, child psychology, mother child relations, school achievement

A depressão é um transtorno mental no qual há significativa alteração do humor ou afeto e está frequentemente associada à incapacitação funcional e a prejuízos na qualidade de vida em razão de sua sintomatologia, caracterizada por tristeza, irritação e/ou sensação de vazio, associadas a alterações somáticas e de pensamento (Associação Americana de Psiquiatria [APA], 2014). Segundo a Organização Mundial da Saúde (OMS, 2012), atualmente é um dos transtornos psiquiátricos mais frequentes, sendo a terceira doença que mais causa incapacidade no mundo. Estima-se que, em 2030, será a primeira causa de incapacitação, afetando a vida social e ocupacional de grande parte da população mundial.

Estudos epidemiológicos em países ocidentais apontam que a prevalência da depressão ao longo da vida na população geral é de $16 \%$, acometendo entre 5,3\% e 6,6\% de pessoas no período de um ano (Hammen, Bistricky, \& Ingran, 2010). Apesar da variabilidade do curso desse transtorno mental, o DSM-5 destaca que alguns indivíduos com depressão raramente permanecem em remissão dos sintomas, podendo apresentar algumas manifestações mais leves entre episódios característicos, sendo que o risco de recorrência e cronicidade

1 Endereço para correspondência: Rua Tenente Catão Roxo, 2650, Monte Alegre, Ribeirão Preto, SP, Brasil. CEP: 14051-140.E-mail: srlourei@ fmrp.usp.br dos episódios se torna maior em função da gravidade da sintomatologia (APA, 2014).

A depressão atinge principalmente as mulheres, com uma proporção duas vezes maior do que os homens, sendo verificada maior vulnerabilidade principalmente no período reprodutivo e maior ocorrência na faixa etária entre 18 e 49 anos, com elevada taxa de episódios moderados ou graves (Andrade et al., 2012). Considerando tais características, destaca-se que a recorrência do transtorno depressivo ao longo da vida pode ter implicações diversas para o exercício da maternidade, para a interação mãe-criança e para o ambiente familiar, já que, na maioria das culturas, a mãe exerce o papel de principal cuidadora dos filhos. Dada a magnitude desse transtorno e a amplitude do seu impacto, justifica-se a relevância de estudos com foco nessa problemática, especialmente quando se trata de mulheres/ mães.

A convivência de crianças com mães que apresentem depressão é considerada, pois, uma condição de adversidade ao desenvolvimento infantil. Destaca-se que, de acordo com o referencial teórico da psicopatologia do desenvolvimento, tal adversidade se associaria a outras condições de vida, as quais se configuram como influências cumulativas para 
desfechos desenvolvimentais, favorecendo a competência e/ ou a disfunção ao longo do ciclo vital (Toth \& Cichetti, 2010).

Sob essa perspectiva, problemas de saúde mental maternos, especificamente a depressão, podem exercer impacto negativo em diversos domínios do funcionamento infantil. Ressaltam-se os problemas de comportamento internalizantes e externalizantes (Bagner, Pettit, Lewinsohn, \& Seeley, 2010; Fanti \& Henrich, 2010), a psicopatologia infantil (Tompson et al., 2010), bem como os prejuízos na regulação emocional (Silk, Shaw, Skuban, Oland, \& Kovacs, 2006), no autoconceito (Mian, Tango, Lopes, \& Loureiro, 2009) e no desempenho escolar das crianças (Silverstein, Augustyn, Cabral, \& Zuckerman, 2006).

O impacto da depressão materna pode ser verificado por meio da avaliação dos indicadores de adaptação das crianças que convivem com tal condição. Entre esses indicadores, destacam-se as tarefas de desenvolvimento definidas como desafios típicos em cada estágio de vida, que demandam enfrentamento para adaptação positiva (Papalia \& Feldman, 2013). Para as crianças em idade escolar, a realização das tarefas próprias da fase se expressa por meio de indicadores comportamentais, de socialização e de aprendizagem, caracterizando, assim, a adaptação às regras da família, do ambiente escolar e da sociedade.

Os problemas de comportamento representam as dificuldades mais frequentes nas crianças em idade escolar. Segundo Achenbach et al. (2008), esses problemas podem ser expressos pelos comportamentos internalizantes, enquanto distúrbios pessoais incluindo ansiedade, retraimento, depressão e sentimento de inferioridade, e por comportamentos externalizantes, que favorecem conflitos com o ambiente, como aqueles que envolvem características de desafio, impulsividade, agressividade e hiperatividade.

A atenção aos indicadores de desempenho escolar nessa faixa etária, que corresponde ao início da escolarização formal e da alfabetização, torna-se uma questão importante. A criança encontra-se voltada à realização de tarefas intelectuais e sociais, experimentando novos aprendizados junto aos pares, à educação formal e à família, que nortearão sua competência e sua capacidade produtiva (Papalia \& Feldman, 2013). De acordo com Campezatto e Nunes (2007), é nessa faixa etária que se identifica maior procura por atendimento clínico especializado de psicologia e psiquiatria para crianças, em função de dificuldades de aprendizagem e/ ou de comportamento, sinalizando a relevância de se colocar em foco essa fase do desenvolvimento.

Faz-se necessário também, nesse período do desenvolvimento, atentar para a questão do sexo na avaliação das tarefas desenvolvimentais, já que tal variável tem se mostrado relevante na prevalência de alguns transtornos mentais. Diversos estudos têm apontado que a exposição ao fator de risco da depressão materna está associada a prejuízos no comportamento e na saúde mental dos filhos, porém com diferentes formas de manifestação dependendo do sexo da criança. Estudos empíricos apontam que os meninos evidenciam mais problemas de comportamento externalizante do que as meninas, que tendem a apresentar mais dificuldades internalizantes (Loosli \& Loureiro, 2010). Esses resultados mostram que a interação mãe depressiva- criança afeta o desenvolvimento infantil, de acordo com o sexo da criança.

Foster et al. (2008) identificaram forte relação entre a duração do episódio depressivo atual da mãe e problemas de comportamento internalizantes para as meninas e externalizantes para os meninos em idade escolar. Os filhos de mães com depressão crônica, por sua vez, apresentaram níveis significativamente maiores de relato parental de problemas de comportamento externalizante em comparação aos filhos de mães sem depressão, sendo detectada diferença entre os sexos, com maior frequência de comportamentos externalizantes para meninos e internalizantes para meninas (Ashman, Dawson, \& Panagiotides, 2008).

Ao analisarem trajetórias de sintomas internalizantes, Jenkins e Curwen (2008) também identificaram associação entre altos níveis de sintomas depressivos maternos e o aumento dos problemas internalizantes para meninas entre 10 e 14 anos de idade, mas com diminuição desses mesmos prejuízos para os meninos, com o passar do tempo. No mesmo sentido, Garai et al. (2009) sugeriram que as meninas expostas à depressão materna estão em situação de maior risco para apresentarem problemas de comportamento internalizantes em comparação aos meninos.

As influências da depressão materna para os meninos, com predomínio de problemas externalizantes, foram abordadas por diversos autores. Shaw, Hyde e Brennan (2012), estudando uma coorte de meninos de baixa renda, verificaram que a exposição à depressão materna entre um ano e meio e dois anos de idade mostrou-se um importante fator de risco para a presença de comportamento antissocial quando os filhos estavam na faixa etária dos 10 aos 17 anos. Gross, Shaw, Burwell e Nagin (2009) relataram que os filhos de mães com trajetória de depressão moderada tendendo a grave foram apontados pelos professores como apresentando significativamente mais problemas externalizantes em comparação aos filhos de mães com trajetória de depressão leve e moderada tendendo a leve. Brown, Barbarin e Scott (2013) estudaram uma ampla amostra de meninos negros e, ao analisarem modelos de risco para sintomas internalizantes, identificaram que a maioria apresentou baixos níveis desses sintomas, independente de conviverem com mães com sintomas depressivos.

Tais achados, no entanto, não são confirmados por outros estudos. Blatt-Eisengart, Drabick, Monahan e Steinberg (2009) detectaram o predomínio de problemas externalizantes para meninas em idade escolar ao investigarem as diferenças entre os sexos na relação entre sintomas depressivos maternos e problemas de comportamento externalizantes de crianças. Ao que se refere aos problemas de comportamento internalizantes, Bureau, Easterbrooks e Lyons-Ruth (2009) não constataram diferenças entre os sexos, aos oito anos de idade das crianças, ao examinarem a relação entre sintomas depressivos maternos e sintomas depressivos das crianças. Cortes, Fleming, Catalano e Brown (2006) também não verificaram diferenças relativas a sintomas depressivos relatados por meninos e meninas em idade escolar, filhos de mães com depressão. Verificaram, contudo, que as diferenças de sexo relativas aos sintomas depressivos começaram a aparecer no início da adolescência, fase na qual as meninas 
mostraram-se mais vulneráveis ao impacto da depressão materna.

Com base em uma revisão da literatura, Loosli e Loureiro (2010) relataram a presença de diferenças entre os sexos em crianças expostas à depressão materna na maioria dos estudos empíricos incluídos. As autoras identificaram estudos em que o predomínio era de problemas de comportamento externalizantes para os meninos no período pré-escolar, além de dificuldades internalizantes para as meninas e externalizantes para os meninos na fase escolar e, na adolescência, predomínio de problemas internalizantes para as meninas.

Em uma metanálise relativa a artigos empíricos sobre a associação entre depressão materna e problemas de comportamento em crianças e adolescentes, Goodman et al. (2011) não identificaram diferenças entre os sexos quanto aos comportamentos externalizantes das crianças. Contudo, constataram uma associação mais forte e significativa entre depressão materna e problemas de comportamento internalizante para as meninas em comparação aos meninos, sendo que tal diferença foi significativa apenas para os estudos com crianças entre seis e 10 anos. Os autores verificaram maior tamanho do efeito para as dificuldades internalizantes quando eram utilizados, nos estudos empíricos, instrumentos diagnósticos para avaliação da depressão materna, amostras clínicas e mães como fonte de informação do comportamento dos filhos. Ressaltaram a presença de possíveis indicadores relativos à hereditariedade, a diferenças na sensibilidade de meninas e meninos frente aos estressores associados à depressão materna e a diferenças nas práticas parentais enquanto condições que poderiam influenciar os distintos desfechos desenvolvimentais de meninos e meninas.

Em relação à literatura nacional, destaca-se o estudo desenvolvido por Bordin et al. (2009), que abordou a questão das diferenças entre os sexos e a saúde mental de crianças expostas a diversos fatores de risco, incluindo sintomas depressivos maternos. O objetivo principal do estudo foi examinar a relação entre exposição à punição física severa e problemas de saúde mental em crianças de seis a 17 anos. Os autores verificaram que os sintomas depressivos e ansiosos das mães foram correlacionados com problemas de comportamento internalizante e externalizante para as crianças, e que as meninas apresentaram maior risco para dificuldades internalizantes do que os meninos. Em tal estudo, foi utilizada uma amostra da comunidade sem a confirmação diagnóstica para a depressão materna, sendo a presença de sintomas depressivos das mães identificada por meio de instrumento de rastreamento.

O desempenho nas tarefas escolares torna-se, nesse contexto, uma questão que merece atenção. Papalia e Feldman (2013) apontaram que as meninas tendem a ter melhor desfecho no contexto acadêmico, especialmente quanto às notas nas diversas matérias, à reprovação e aos problemas escolares em geral. Nesse sentido, Bandeira, Rocha, Souza, Del Prette, e Del Prette (2006) identificaram, nos meninos, maior frequência de problemas de comportamento em geral em comparação às meninas, em uma amostra de crianças de $1^{\mathrm{a}}$. a $4^{\mathrm{a}}$. série do ensino fundamental. Verificaram também que a maior frequência de comportamentos problemáticos estava associada a maiores dificuldades de aprendizagem, a menor nível de habilidades sociais e a menor grau de competência acadêmica por parte das crianças.

Marturano, Toller e Elias (2005), em uma amostra de crianças com rendimento escolar pobre, não identificaram diferenças entre sexos quanto ao desempenho escolar. Constataram, no entanto, mais problemas internalizantes para as meninas e não encontraram diferenças de sexo quanto aos comportamentos externalizantes. Vale ressaltar que não foram encontrados estudos que incluíssem o contexto de depressão materna associado à avaliação do desempenho escolar considerando-se o sexo das crianças.

Ao se analisar a literatura, foram identificadas peculiaridades e uma diversidade de achados quanto ao perfil de meninos e meninas que convivem com a depressão materna, fato que sinaliza a necessidade de novos estudos que abordem essa questão, em concordância com Goodman et al. (2011). Ampliando essas proposições, sob a perspectiva da psicopatologia do desenvolvimento, o presente estudo incluiu como medida de adaptação também os indicadores relativos ao desempenho escolar, enquanto tarefa típica e relevante desse período do desenvolvimento. Pretende-se, assim, contribuir para a compreensão quanto às diferenças comportamentais e de desempenho escolar de meninos e meninas expostos à depressão materna, o que potencialmente contribui para a proposição de práticas de saúde mental.

Nesse contexto, insere-se o objetivo do presente estudo: comparar as diferenças de sexo relativas ao comportamento e ao desempenho escolar de crianças em idade escolar que convivem com a depressão materna recorrente, correlacionando os desfechos comportamentais e escolares. As hipóteses norteadoras foram as seguintes: os meninos que convivem com a depressão materna apresentarão mais problemas externalizantes; as meninas apresentarão mais comportamentos internalizantes; e as crianças com dificuldades comportamentais apresentarão prejuízo no desempenho escolar, independente do sexo.

\section{Método}

O presente estudo foi apreciado e aprovado por Comitê de Ética e a participação das mães, assim como das crianças, foi voluntária mediante a assinatura do Termo de Consentimento Livre e Esclarecido. Adotou-se um delineamento de comparação entre grupos distribuídos pelo sexo e correlacional quanto às variáveis comportamento e desempenho escolar das crianças ${ }^{1}$.

\section{Participantes}

Participaram do estudo 40 díades mãe-criança provenientes de uma amostra clínica de conveniência. As crianças contavam com idades entre sete e 12 anos e residiam

1 O presente trabalho é derivado da Dissertação de Mestrado de Lívia Loosli, defendida em 2011, junto à Faculdade de Medicina de Ribeirão Preto - Universidade de São Paulo, e decorre de um projeto mais amplo que vem estudando o impacto da depressão materna e variáveis diversas do contexto familiar para desfechos desenvolvimento de crianças em idade escolar, sob orientação da Prof. ${ }^{a}$ Dr. ${ }^{a}$ Sonia Regina Loureiro. 
com suas mães biológicas, cujas idades variavam entre 25 e 45 anos e que apresentavam história clínica de Transtorno Depressivo Recorrente, com diagnóstico sistematicamente confirmado por meio de entrevista clínica estruturada, de acordo com os critérios da Classificação Estatística Internacional de Doenças e Problemas Relacionados à Saúde-CID 10 (OMS, 1989/2014). Foram incluídas mães com pelo menos um episódio depressivo moderado ou grave nos últimos dois anos e sem episódios nos últimos seis meses, de modo a minimizar a interferência da sintomatologia atual no relato sobre as crianças.

As crianças foram identificadas a partir de suas mães, sendo distribuídas pelo sexo em dois grupos: G1 - 20 meninos e G2 - 20 meninas. Os critérios de exclusão para o estudo foram: crianças órfãs ou com história de adoção, com deficiência intelectual ou com deficiências sensoriais aparentes. Foram excluídas mães que apresentaram diagnóstico de Transtorno Afetivo Bipolar, Depressão com episódio único, Transtorno Depressivo Recorrente com episódios leves e com comorbidade de outros transtornos, tais como: Esquizofrenia, Transtorno Esquizoafetivo, Transtornos de Personalidade e uso/abuso de substâncias psicoativas.

A seleção das mães foi realizada tendo por base informações de 2.626 prontuários de dois serviços de saúde mental da rede municipal de uma cidade de médio porte do interior do estado de São Paulo, sendo que 699 (26,61\%) eram de mulheres com o diagnóstico de Depressão Recorrente ou Depressão Maior moderada ou grave, e 122 (17,45\%) tinham idades entre 25 e 45 anos e eram mães com filhos em idade escolar. Dos 122 prontuários identificados foram excluídas 19 mulheres com apenas um episódio de Depressão Maior e 27 mulheres que apresentaram sintomas moderados ou graves nos últimos seis meses; 19 mães não foram localizadas e 12 não aceitaram participar. As 45 possíveis participantes foram convidadas a responderem uma entrevista clínica estruturada para confirmação diagnóstica e, com base nos critérios de exclusão acima mencionados, foram incluídas 40 mães.

\section{Instrumentos e Materiais}

\section{Para a seleção dos participantes.}

Com as mães. Entrevista Clínica Estruturada para o DSM-IV (SCID), cuja tradução e adaptação para o nosso meio conduzida por Del-Ben et al. (2001) apresenta qualidades psicométricas satisfatórias, sendo considerada padrão ouro em estudos clínicos como medida de saúde mental que permite a classificação diagnóstica de transtornos psiquiátricos. Foram utilizados: Revisão Geral, SCID I (módulos A, B, C, D) e revisão geral da SCID II para confirmação diagnóstica de Transtorno Depressivo Recorrente das mães e exclusão de comorbidades. Destaca-se que a quinta edição do Manual Diagnóstico e Estatístico de Transtornos Mentais (DSM-5; APA, 2014) manteve os critérios diagnósticos relativos aos Transtornos Depressivos apresentados na versão anterior, utilizada para a elaboração da SCID.

Com as crianças. Matrizes Progressivas Coloridas de Raven - Escala Especial. Trata-se de um teste intelectual não verbal desenvolvido para avaliação da inteligência geral de crianças na faixa etária dos cinco aos 11 anos, tendo padronização brasileira (Angelini, Alves, Custódio, Duarte, \& Duarte, 1999). Quanto à precisão do instrumento, o coeficiente de correlação entre os itens (método das metades) para a amostra total foi de 0,92 (Angelini et al., 1999). O instrumento foi utilizado para a exclusão de crianças com comprometimento intelectual.

\section{Para o estudo propriamente dito.}

Com as mães. Questionário de Capacidades e Dificuldades (SDQ), elaborado por Goodman (1997) e traduzido e adaptado às características socioculturais brasileiras por Fleitlich, Córtazar e Goodman (2000). Trata-se de um questionário de rastreamento de problemas de saúde mental infantil por meio da avaliação do comportamento da criança nos últimos seis meses. Apresenta versões para pais ou responsáveis, professores, crianças e adolescentes de quatro a 16 anos, sendo de uso livre e disponível para acesso no site: www.sdqinfo.com.

Para o estudo, foi utilizada a versão para pais ou responsáveis, respondida apenas pelas mães. O instrumento é composto por 25 itens distribuídos igualmente em cinco escalas de cinco itens cada uma, sendo quatro relativas a dificuldades - Sintomas Emocionais, Problemas de Conduta, Hiperatividade, Problemas de Relacionamento com Colegas - e uma relativa a capacidades - Comportamento Pró-Social.

As propriedades psicométricas do SDQ foram descritas por Woerner et al. (2004), que apontaram resultados com diferenças significativas $(p<0,001)$ para as versões de pais e professores por meio da comparação de médias de amostras clínicas com não-clínicas. Na comparação do SDQ com o Development and Well-Being Assessment for Children and Adolescents (DAWBA), foram identificados bons índices de concordância entre os dois instrumentos $\left(\chi^{2}=13,1\right.$; $p<0,001)$. Os estudos de fidedignidade do SDQ utilizaram o método teste-reteste e o cálculo do alfa de Cronbach, não sendo observada diferença significativa entre a primeira e a segunda avaliação (teste de amostras pareadas, $p=0,148$ ). A correlação entre as duas avaliações foi 0,79 , enquanto a correlação intraclasse foi 0,77 (ambos com $p<0,001$ ), valores considerados satisfatórios.

Utilizou-se, ainda, uma ficha sociodemográfica, elaborada para a finalidade do estudo. Foi empregada para o levantamento de informações sociodemográficas dos participantes, incluindo idade, escolaridade e sexo das crianças, além de idade, escolaridade e ocupação das mães, bem como classe socioeconômica das famílias.

Com as crianças. Teste de Desempenho Escolar (TDE), padronizado por Stein (1994). Tem por finalidade avaliar as capacidades fundamentais para o desempenho escolar de crianças da $1^{\mathrm{a}}$. a $6^{\mathrm{a}}$. série do ensino fundamental, sendo composto por três subtestes: Escrita, Aritmética e Leitura, com normas nacionais para escolaridade e idade. As propriedades psicométricas do TDE foram verificadas em estudo realizado na cidade de Porto Alegre - RS. Os coeficientes de correlação entre os subtestes e o teste total mostraram-se satisfatórios, variando de 0,67 a 0,95 (todos 
com $\mathrm{p}<0,001)$ e o coeficiente alfa variou de 0,93 a 0,98 , considerando-se os três subtestes e o teste total (Stein, 1994).

\section{Procedimento}

A coleta de dados foi realizada em sessões individuais nos serviços de Saúde Mental nos quais as mães foram identificadas ou em suas próprias residências, buscandose preservar as condições de privacidade e conforto. As avaliações ocorreram após a apresentação dos objetivos do estudo e a assinatura do Termo de Consentimento, sendo realizadas por três psicólogas e duas estudantes de psicologia previamente treinadas para a aplicação dos instrumentos, que foram utilizados de acordo com as recomendações técnicas específicas.

Avaliação com as mães. Foi realizado o preenchimento da ficha sociodemográfica e a aplicação da SCID para a confirmação diagnóstica. Quando confirmado o diagnóstico de depressão recorrente, as mães responderam ao SDQ. Em geral, a avaliação foi realizada em uma única sessão, com duração aproximada de 90 minutos.

Avaliação com as crianças. Após um breve rapport foi aplicado o Raven. Em seguida, as crianças responderam ao TDE. A avaliação foi realizada em uma única sessão individual, face a face, com duração média de 50 minutos.

\section{Análise dos Dados}

Os instrumentos foram codificados de acordo com as recomendações técnicas específicas e os resultados obtidos foram comparados aos dados normativos de cada um. A ficha sociodemográfica foi quantificada sob a forma de frequência e porcentagem. Os resultados do Raven foram transformados em percentil, sendo considerado critério para a inclusão no estudo percentil $>25$.

Em relação ao $\mathrm{SDQ}$, os escores parciais e total são classificados como normal, limítrofe ou anormal e cada escala apresenta notas de corte para tal classificação. Quanto ao escore total, adotou-se como nota de corte a pontuação maior que 16 como indicativa de dificuldades comportamentais (Fleitlich et al. 2000). Para os objetivos deste estudo, as classificações normal e limítrofe foram agrupadas como Sem Dificuldade (SD) e a classificação anormal foi considerada como Com Dificuldade (CD) quanto ao comportamento.

Quanto ao TDE, de acordo com as normas para escolaridade e/ou idade, os escores são classificados como superior, médio ou inferior. Para este estudo, as classificações superior e média foram agrupadas sendo consideradas como $\mathrm{SD}$ e a classificação inferior $\mathrm{CD}$ quanto ao desempenho escolar.

Inicialmente, foram realizadas análises descritivas a fim de caracterizar o perfil dos participantes quanto a características demográficas, utilizando-se medidas de frequência e porcentagem. Para a análise dos dados, procedeu-se à comparação entre os grupos diferenciados pelo sexo sendo utilizados o Teste Não-Paramétrico de Mann Whitney, Teste do Qui-Quadrado, Teste Exato do Qui-quadrado e Teste Exato de Fisher, dependendo da normalidade da distribuição das variáveis. Procedeu-se à análise de regressão logística bivariada com o objetivo de se examinar possíveis influências de variáveis sociodemográficas nos resultados obtidos. Para verificar as associações significativas entre o comportamento e o desempenho escolar das crianças, foi utilizada a correlação não paramétrica de Spearman. Adotouse, em todas as análises, o nível de significância de $\mathrm{p} \leq 0,05$.

\section{Resultados}

Em relação ao perfil demográfico e cognitivo das crianças, as meninas apresentaram média de idade de 10,15 anos $(D P=1,78)$ e os meninos, média de 10,32 anos $(D P=1,67)$. Quanto à escolaridade, $55 \%$ dos meninos e das meninas cursavam entre a $1^{\mathrm{a}}$. e a $4^{\mathrm{a}}$. série, com média de 4,05 anos de estudo $(D P=1,67)$. Em relação ao nível cognitivo, avaliado por meio do Raven, a média do percentil obtido pelos meninos e meninas foi, respectivamente, $69,05(D P=20,18)$ e 58,85 $(D P=25,09)$. Com base em análise estatística, não foram observadas diferenças significativas quanto às variáveis acima descritas.

Quanto às mães, as do grupo de meninos apresentaram média de idade de 33,7 anos $(D P=3,46)$, enquanto as mães das meninas apresentaram média de 38,5 anos $(D P=5,43)$. No que se refere à escolaridade, a média de anos de estudo para as mães do grupo de meninos foi de 9,20 $(D P=2,23)$ e as mães das meninas tiveram média de 6,75 anos de estudo $(D P=2,19)$. De acordo com análise estatística, observou-se que as mães de meninas tiveram média de idade maior e menos anos de estudo do que as mães dos meninos, com diferença estatisticamente significativa $(p \leq 0,05)$. Em relação à ocupação, $45 \%$ das mães de meninos e 55\% das mães de meninas trabalhavam e recebiam salário, e não foram observadas diferenças significativas quanto a essa variável. Predominaram nos dois grupos, as configurações familiares com mãe, pai ou padrasto e até três filhos, com poder aquisitivo corresponde à Classe $\mathrm{C}$, de acordo com a Associação Brasileira de Empresas de Pesquisa (ABEP, 2008).

A Tabela 1 apresenta os dados referentes ao comportamento das crianças com as comparações entre os sexos, segundo as escalas do SDQ. Observou-se diferença estatisticamente significativa entre sexos na Escala de Sintomas Emocionais, segundo a qual $80 \%$ das meninas apresentaram indicadores de dificuldades em comparação a 50\% dos meninos. Quanto ao escore da Escala de Sintomas Emocionais, as meninas apresentaram pontuação média maior que quatro, nota de corte indicativa de dificuldades dessa escala, com diferença significativa em comparação ao escore médio apresentado pelos meninos.

Em relação ao Total de Dificuldades, 42,5\% das crianças apresentaram dificuldades comportamentais, sendo 35\% dos meninos e metade das meninas. De acordo com a pontuação do escore total, as meninas apresentaram média maior do que a dos meninos, contudo não se observou diferença significativa entre os sexos. A pontuação média maior que 16 , nota de corte segundo o critério do instrumento, é considerada como indicativa de dificuldades comportamentais, e tal dado foi observado para o grupo de meninas. Quanto às escalas 
Tabela 1. Avaliação do comportamento das crianças por meio do SDQ comparado entre os sexos.

\begin{tabular}{|c|c|c|c|c|c|c|}
\hline \multirow[t]{2}{*}{ SDQ } & \multicolumn{2}{|c|}{$\begin{array}{c}\text { Meninos } \\
\mathrm{N}_{1}=\mathbf{2 0}\end{array}$} & \multicolumn{2}{|c|}{$\begin{array}{c}\text { Meninas } \\
\mathrm{N}_{2}=\mathbf{2 0}\end{array}$} & \multirow{2}{*}{$\begin{array}{l}\text { Total } \\
\mathrm{N}=40 \\
\mathrm{n}(\%)\end{array}$} & \multirow[t]{2}{*}{$p$} \\
\hline & n (\%) & M (DP) & n (\%) & M (DP) & & \\
\hline \multicolumn{7}{|c|}{ Total Dificuldades } \\
\hline $\mathrm{SD}$ & $13(65)$ & & $10(50)$ & & $23(57,5)$ & \multirow{2}{*}{0,330} \\
\hline $\mathrm{CD}$ & $07(35)$ & & $10(50)$ & & $17(42,5)$ & \\
\hline Escore & & $13,5(5,95)$ & & $17(7,54)$ & & 0,140 \\
\hline \multicolumn{7}{|c|}{ Sintomas Emocionais } \\
\hline $\mathrm{SD}$ & $10(50)$ & & $04(20)$ & & $14(35)$ & \multirow{2}{*}{$0,040^{*}$} \\
\hline $\mathrm{CD}$ & $10(50)$ & & $16(80)$ & & $26(65)$ & \\
\hline Escore & & $3,9(2,82)$ & & $6,35(2,39)$ & & $0,005 * *$ \\
\hline \multicolumn{7}{|c|}{ Problemas Conduta } \\
\hline SD & $14(70)$ & & $13(65)$ & & $27(67,5)$ & \multirow{2}{*}{0,730} \\
\hline $\mathrm{CD}$ & $06(30)$ & & $07(35)$ & & $13(32,5)$ & \\
\hline Escore & & $2,65(1,84)$ & & $3,05(2,48)$ & & 0,780 \\
\hline \multicolumn{7}{|l|}{ Hiperatividade } \\
\hline $\mathrm{SD}$ & $13(65)$ & & $15(75)$ & & $28(70)$ & \multirow{2}{*}{0,490} \\
\hline $\mathrm{CD}$ & $07(35)$ & & $05(25)$ & & $12(30)$ & \\
\hline Escore & & $4,2(3,18)$ & & $4,95(3,15)$ & & 0,520 \\
\hline \multicolumn{7}{|c|}{ Prob. Relac. Colegas } \\
\hline SD & $14(70)$ & & $13(65)$ & & $27(67,5)$ & \multirow{2}{*}{0,740} \\
\hline $\mathrm{CD}$ & $06(30)$ & & $07(35)$ & & $13(32,5)$ & \\
\hline Escore & & $2,75(2,12)$ & & $2,65(1,69)$ & & 0,950 \\
\hline \multicolumn{7}{|c|}{ Comport. Pró-social } \\
\hline SD & $20(100)$ & & $20(100)$ & & $40(100)$ & \multirow{2}{*}{1,000} \\
\hline $\mathrm{CD}$ & $00(00)$ & & $00(00)$ & & $00(00)$ & \\
\hline Escore & & $8,15(1,81)$ & & $8,65(1,42)$ & & 0,390 \\
\hline
\end{tabular}

Nota: SD - Sem dificuldade, CD - Com dificuldade; Teste do Qui-Quadrado; Teste Não-Paramétrico de Mann-Whitney; * $p \leq 0,05 ; * * p \leq 0,01$.

de Problemas de Conduta, Hiperatividade, Problemas de Relacionamento com os Colegas e Comportamento Prósocial, não foram observadas diferenças significativas entre os grupos de meninos e meninas, sendo que a maioria das crianças não apresentou dificuldades nessas escalas.

Uma vez que as mães das meninas apresentaram menor escolaridade e maior idade em comparação às mães dos meninos, verificou-se se as diferenças significativas entre os sexos observadas quanto ao comportamento das crianças poderiam ter sido influenciadas por tais variáveis. A Tabela 2 apresenta a análise de acordo com a correção de tais variáveis, ajustando-se o escore total da Escala de Sintomas Emocionais do SDQ pela escolaridade e idade maternas, considerando-se o sexo das crianças. A análise de regressão logística bivariada revelou a presença de diferença significativa na escala de Sintomas Emocionais. Esse resultado indicou que as meninas apresentaram escore total maior nessa escala em comparação aos meninos, independentemente da escolaridade ou da idade maternas.

O desempenho escolar de meninos e meninas também foi avaliado e não foram identificadas diferenças significativas entre os grupos separados pelo sexo. Quanto ao escore total, pouco mais da metade das crianças $(55 \%)$ apresentou dificuldades relativas ao desempenho escolar, sendo a maioria de meninas (60\%) e metade dos meninos. Considerando-se as habilidades específicas, as maiores dificuldades das crianças

Tabela 2. Análise de regressão logística bivariada, tendo como variáveis independentes a escolaridade e idade maternas e o escore total da Escala de Sintomas Emocionais do SDQ, e como variável dependente o sexo das crianças $(n=40)$.

\begin{tabular}{lllc}
\hline \multicolumn{1}{c}{ Variáveis } & $\boldsymbol{p}^{*}$ & OR & IC (95\%) \\
\hline Escala de Sintomas Emocionais - Escore Total & $0,040^{*}$ & 1,38 & $1,009-1,876$ \\
Escolaridade mãe & $0,010^{*}$ & 0,64 & $0,443-0,918$ \\
Escala de Sintomas Emocionais - Escore Total & $0,040^{*}$ & 1,34 & $1,004-1,793$ \\
Idade mãe & $0,020^{*}$ & 1,20 & $1,030-1,409$ \\
\hline
\end{tabular}

Nota: $* p \leq 0,05$. 
foram observadas em relação à aritmética $(57,5 \%)$ e à escrita $(47,5 \%)$. Com relação à leitura, as crianças apresentaram um melhor desempenho.

A Tabela 3 apresenta os dados referentes às correlações evidenciadas entre o desempenho escolar e as dificuldades comportamentais. Essa análise foi feita apenas para o grupo das meninas, já que para os meninos não foram verificadas correlações entre tais variáveis. Pode-se observar que os problemas comportamentais de relacionamento com os colegas foram correlacionados negativamente com o desempenho escolar total e com os domínios de leitura e escrita. Tais dados sugerem que as meninas que apresentaram mais dificuldades de relacionamento com colegas, também evidenciaram um desempenho escolar mais negativo, principalmente relacionado às habilidades de escrita $\mathrm{e}$ leitura. Também foi identificada correlação negativa entre as dificuldades relativas aos sintomas emocionais e o desempenho escolar no domínio da escrita, sugerindo que
(2010), a partir de uma revisão sistemática da literatura, e achados de estudos empíricos internacionais (Foster et al., 2008; Garai et al., 2009; Jenkins \& Curwen, 2008). Tais problemas emocionais, avaliados pelas mães no presente estudo, referem-se principalmente a queixas somáticas e de insegurança, próprias de problemas de comportamento internalizante, o que se assemelha aos resultados de Jenkins e Curwen (2008) com base em dados coletados com as próprias crianças.

Foi verificado, por outro lado, que os meninos não apresentaram mais problemas de comportamento externalizante em comparação às meninas, o que se mostrou discordante dos estudos internacionais de Foster et al. (2008), Shaw et al. (2012) e Gross et al. (2009), conduzidos com populações específicas e distintas, tais como mães com depressão de maior gravidade, famílias com baixa renda e etnia negra, respectivamente. As diferenças metodológicas relativas ao número de participantes e idade dos mesmos,

Tabela 3. Correlações significativas entre o desempenho escolar (TDE) e as dificuldades comportamentais (SDQ) para o grupo de meninas

\begin{tabular}{ccccc}
\hline Grupo & \multicolumn{2}{c}{ Variáveis } & $\boldsymbol{r}$ & $\boldsymbol{p}$ \\
Meninas & Desempenho Escolar (TDE) & Comportamento (SDQ) & $0,010^{* *}$ \\
\hline Total $\downarrow$ & Probl. Relacionamento $\uparrow$ & $-0,55$ & $0,030^{*}$ \\
& Leitura $\downarrow$ & Probl. Relacionamento $\uparrow$ & $-0,48$ & $0,008^{* *}$ \\
Escrita $\downarrow$ & Probl. Relacionamento $\uparrow$ & $-0,57$ & $0,010^{* *}$ \\
\hline Escrita $\downarrow$ & Sint. Emocionais $\uparrow$ & $-0,53$ & \\
\hline
\end{tabular}

Nota: ${ }^{*} p \leq 0,05 ; * * p \leq 0,01$; correlação não paramétrica de Spearman; $\uparrow=$ aumentado; $\downarrow=$ diminuído

as meninas que apresentaram mais problemas emocionais evidenciaram também mais dificuldades de escrita.

\section{Discussão}

O principal objetivo do estudo foi comparar grupos distribuídos pelo sexo, considerando-se o comportamento e o desempenho escolar de crianças que convivem com mães com depressão recorrente. Destaca-se inicialmente que, com base na análise estatística relativa ao perfil sociodemográfico e cognitivo das crianças, não foram identificadas diferenças significativas entre os grupos, os quais eram homogêneos em relação a tais variáveis, sendo que as diferenças quanto ao perfil comportamental e de desempenho escolar não foram explicadas por tais indicadores. Todavia, indicadores relativos a características demográficas das mães apresentaram diferenças estatisticamente significativas, sendo que as mães das meninas tiveram média de idade maior e menos anos de estudo. Essas diferenças foram analisadas por meio de teste estatístico, permitindo verificar que tais peculiaridades da amostra não influenciaram os resultados referentes às dificuldades comportamentais das crianças, especialmente quanto ao predomínio de problemas internalizantes para as meninas.

O comportamento dos meninos foi comparado em relação ao das meninas. Os resultados mostraram que as meninas apresentaram mais dificuldades relativas apenas aos sintomas emocionais, o que reitera considerações de Loosli e Loureiro as fontes e peculiaridades das amostras, os informantes e os instrumentos utilizados podem justificar as divergências entre os resultados do presente trabalho e dos estudos acima referidos.

Os dados do presente estudo são concordantes com os resultados do trabalho desenvolvido por Bordin et al. (2009) em nosso meio, que identificaram associação entre sintomas depressivos e ansiosos das mães e problemas internalizantes para as meninas. $\mathrm{O}$ estudo de Bordin et al. utilizou, contudo, uma amostra da comunidade em situação de risco psicossocial e não realizou confirmação diagnóstica para a depressão materna.

Os resultados do presente estudo vão na mesma direção da metanálise de Goodman et al. (2011), que apontou que a depressão materna foi mais fortemente associada a problemas internalizantes em meninas do que em meninos entre seis e 10 anos. Os achados da metanálise de Goodman et al., bem como os dados obtidos por Bordin et al. (2009), não identificaram diferenças entre os sexos quanto aos problemas externalizantes de comportamento.

O desempenho escolar das crianças também foi avaliado. Não foram observadas diferenças significativas entre meninos e meninas, considerando-se o escore total e os domínios específicos de aritmética, escrita e leitura. Destacase que não foram identificados estudos empíricos que avaliaram o desempenho escolar de crianças que conviviam com a depressão materna e que compararam meninos e meninas. No contexto geral e de outras condições adversas, a ausência de diferenças significativas entre os sexos quanto 
ao desempenho acadêmico foi observada por Marturano et al. (2005), o que pode indicar que a influência de condições familiares adversas pouco se expressa na comparação de dificuldades escolares entre meninos e meninas.

Outro objetivo deste estudo foi correlacionar os dois domínios das tarefas de desenvolvimento típicas da idade escolar: o comportamento e o desempenho escolar das crianças expostas à depressão materna recorrente. Quanto ao grupo de meninos, não foram identificadas correlações entre o comportamento e o desempenho escolar. Em relação às meninas foram observadas correlações negativas entre problemas emocionais e habilidades de escrita, assim como entre problemas de relacionamento com colegas e dificuldades escolares no total e nos domínios de leitura e escrita. Na mesma direção de tais achados, com crianças de $1^{\mathrm{a}}$. a $4^{\mathrm{a}}$. série do ensino fundamental, Bandeira et al. (2006) também identificaram que, quanto menor o nível de habilidades sociais, mais comportamentos problemáticos, maiores dificuldades de aprendizagem e menor grau de competência acadêmica foram apresentados pelas crianças.

A principal contribuição do presente estudo consiste na identificação, para crianças em idade escolar que convivem com mães com depressão recorrente, de diferenças entre os sexos quanto aos problemas de comportamento internalizante, sendo as meninas mais vulneráveis a tais problemas, incluindo também dificuldades de desempenho escolar. Estes resultados reiteram os indicadores epidemiológicos quanto à maior incidência de sintomas depressivos em mulheres, contemplados pelos sintomas emocionais aqui avaliados, e sinalizam uma maior influência da psicopatologia materna para as filhas, resultado que sugere uma possível transmissão intergeracional. $\mathrm{Na}$ perspectiva da psicopatologia do desenvolvimento, destacase que a vulnerabilidade relacionada ao sexo associa-se à depressão materna recorrente, enquanto condição adversa ao desenvolvimento infantil, favorecendo desfechos particulares quanto ao comportamento internalizante em meninas, o que pode se relacionar a outros indicadores de (des)adaptação de escolares em suas tarefas desenvolvimentais típicas.

Faz-se necessário destacar algumas limitações inerentes ao delineamento utilizado. O pequeno número de participantes da amostra limita a generalização dos resultados. O desenho transversal do estudo não permitiu investigar associações entre depressão materna e desfechos desenvolvimentais infantis, na comparação entre os sexos, em momentos distintos do ciclo vital dos filhos. Outros limites a serem considerados dizem respeito a não inclusão de outras variáveis do contexto mais amplo, tais como o ambiente familiar, o social e o escolar, já que estas podem agir como fatores de proteção ou risco à situação de adversidade da depressão materna. A utilização de um único informante também pode introduzir um viés, já que o comportamento infantil foi avaliado pelo relato das mães, não sendo incluídas outras fontes de informação tais como professores e pais.

Apesar de tais limitações, alguns pontos positivos merecem ser destacados. Considera-se que o presente estudo contribuiu para o avanço do conhecimento na área da saúde mental materna e infantil. $O$ estudo atendeu as recomendações metodológicas da metanálise de Goodman et al. (2011) quanto à relevância da precisão diagnóstica, uma vez que selecionou uma amostra clínica a partir de criteriosa avaliação por meio de entrevista clínica estruturada, considerada padrão ouro para classificação diagnóstica de transtornos mentais. Foi constituído um grupo homogêneo de mães com depressão recorrente, não sendo incluídas participantes com outras comorbidades psiquiátricas ou com apenas sintomas depressivos leves, o que poderia interferir na interpretação dos resultados. Além disso, apenas mães não sintomáticas por um período mínimo de seis meses forneceram informações sobre o comportamento de seus filhos, fator que contribuiu para minimizar uma possível distorção no relato materno em função da influência de sintomas depressivos.

Os dados do presente estudo apontaram para uma situação de maior vulnerabilidade por parte das meninas, em comparação aos meninos, quanto a problemas emocionais relativos a queixas somáticas e de insegurança, próprias de comportamentos internalizantes, frente à situação de exposição à depressão materna recorrente. Os meninos denotaram maior preservação nos domínios comportamentais e de socialização, resultado que sugere que outras condições contextuais podem estar se configurando como fatores de risco e proteção e influenciando o comportamento dessas crianças. Tais dados sinalizam para a demanda de novos estudos a fim de se verificar a influência de outras variáveis contextuais do ambiente familiar, escolar e social, de forma a identificar condições relevantes, associadas à depressão materna, enquanto riscos cumulativos mediadores dos desfechos desenvolvimentais de escolares. Recursos pessoais e dos ambientes de convívio, por sua vez, podem também se configurar como mecanismos de proteção, favorecedores de resiliência.

Ressalta-se também a necessidade de novas pesquisas que incluam outras fontes de informação além do relato materno, e também estudos com delineamento longitudinal que considerem as diferenças entre os sexos nas diversas fases de desenvolvimento. É importante também investigar a atuação dos mecanismos de resiliência para o enfrentamento da depressão materna e diferenciar variáveis por sexo e etapa de desenvolvimento das crianças.

Os dados deste estudo trazem implicações e contribuições para a prática clínica, no sentido de ressaltar a importância do estabelecimento e definição de políticas públicas de assistência à saúde mental infantil. Para tal, destaca-se a necessidade do foco na prevenção e promoção da saúde mental das mães, além da avaliação e identificação dos prejuízos apresentados pelas crianças expostas à depressão materna. É de suma importância o estabelecimento de estratégias de intervenção adequadas e diferenciadas para meninos e meninas que focalizem as tarefas típicas de desenvolvimento, com especial atenção para as meninas em idade escolar. 


\section{Referências}

Achenbach, T. M., Becker, A, Döpfner, M., Heiervang, E., Roessner, V., Steinhausen, H. C., \& Rothenberger, A. (2008). Multicultural assessment of child and adolescent psychopathology with ASEBA and SDQ instruments: Research findings, applications, and future directions. The Journal of Child Psychology and Psychiatric, 49(3), 251-275.

Andrade, L. H., Wang, Y. P., Andreoni, S., Silveira, C. M., Alexandrino-Silva, C., Siu, E. R., ... Viana, M. C. (2012). Mental disorders in megacities: Findings from the Sao Paulo megacity mental health survey, Brazil. Plos One, 7(2), e31879.

Angelini, A. L., Alves, I. C. B., Custódio, E. M., Duarte, W. F., \& Duarte, J. L. M. (1999). Manual Matrizes Progressivas Coloridas de Raven - Escala Especial. São Paulo: Centro Editor de Testes e Pesquisas em Psicologia.

Ashman, S. B., Dawson, G., \& Panagiotides, H. (2008). Trajectories of maternal depression over 7 years: Relations with child psychophysiology and behavior and role of contextual risks. Development and Psychopathology, 20(1), 55-77.

Associação Americana de Psiquiatria. (2014). Manual Diagnóstico e Estatístico de Transtornos Mentais: DSM-5. Porto Alegre: Artmed.

Associação Brasileira de Empresas de Pesquisa. (2008). Critério de classificação econômica Brasil. Dados com base no Levantamento socio econômico de 2005 -IBOPE. Recuperado de http://www.abep.org/novo/Content.aspx?ContentID=302

Bagner, D. M., Pettit, J. W., Lewinsohn, P. M., \& Seeley, J. R. (2010). Effect of maternal depression on child behavior: A sensitive period? Journal of the American Academy of Child \& Adolescent Psychiatry, 49(7), 699-707.

Bandeira, M., Rocha, S. S., Souza, T. M. P., Del Prette, Z. A. P., \& Del Prette, A. (2006). Comportamentos problemáticos em estudantes do ensino fundamental: Características da ocorrência e relação com habilidades sociais e dificuldades de aprendizagem. Estudos de Psicologia, 11(2), 199-208.

Blatt-Eisengart, I., Drabick, D. A. G., Monahan, K. C., \& Steinberg, L. (2009). Sex differences in the longitudinal relations among family risk factors and childhood externalizing symptoms. Developmental Psychology, 45(2), 491-502.

Bordin, I. A., Duarte, C. S., Peres, C. A., Nascimento, R., Curto, B. M., \& Paula, C. S. (2009). Severe physical punishment: Risk of mental health problems for poor urban children in Brazil. Bulletin of World Health Organization, 87, 336-344.

Brown, J., Barbarin, O., \& Scott, K. (2013). Socioemotional trajectories in black boys between kindergarten and the fifth grade: The role of cognitive skills and family in promoting resiliency. American Journal of Orthopsychiatry, 83(2), 176184.

Bureau, J. F., Easterbrooks, M. A., \& Lyons-Ruth, K. (2009). Maternal depressive symptoms in infancy: Unique contribution to children's depressive symptoms in childhood and adolescence? Development and Psychopathology, 21(2), 519-37.

Campezatto, P. von M., \& Nunes, M. L. T. (2007). Caracterização da clientela das clínicas-escola de cursos de Psicologia da região metropolitana de Porto Alegre. Psicologia: Reflexão e Crítica, 20(3), 376-388.
Cortes, R. C., Fleming, C. B., Catalano, R. F., \& Brown, E. C. (2006). Gender differences in the association between maternal depressed mood and child depressive phenomena from grade 3 through grade 10. Journal of Youth and Adolescence, 35(5), 815-826.

Del-Ben, C. M., Vilela, J. A. A., Crippa, J. A. S., Hallak, J. E. C., Labate, C. M., \& Zuardi, A. W. (2001). Confiabilidade da "Entrevista Clínica Estruturada para o DSM-IV - Versão Clínica" traduzida para o português. Revista Brasileira de Psiquiatria, 23(3), 156-159.

Fanti, K.A. \& Henrich, C.C. (2010). Trajectories of pure and cooccurring internalizing and externalizing problems from age 2 to age 12: Findings from the National Institute of Child Health and Human Development Study of Early Child Care. Developmental Psychology, 46(5), 1159-1175.

Fleitlich, B., Córtazar, P. G., \& Goodman, R. (2000). Questionário de Capacidades e Dificuldades (SDQ). Infanto - Revista de Neuropsiquiatria da Infância e Adolescência, 8(1), 44-50.

Foster, C. E., Webster, M. C., Weissman, M. M., Pilowsky, D. J., Wickramaratne, P. J., Rush, A. J., . . King, C. A. (2008). Course and severity of maternal depression: Associations with family functioning and child adjustment. Journal of Youth and Adolescence, 37(8), 906-916.

Garai, E. P., Forehand, R. L., Colletti, C. J. M., Reeslund, K., Potts, J., \& Compas, B. (2009). The relation of maternal sensitivity to children's internalizing and externalizing problems within the context of maternal depressive symptoms. Behavior Modification, 33(5), 559-582.

Goodman, R. (1997). The Strengths and Difficulties Questionnaire: A research note. Journal of Child Pshicology and Psyquiatry, 38(5), 581-586.

Goodman, S. H., Rouse, M. H., Connell, A.M., Broth, M. R., Hall, C. M., \& Heyward, D. (2011). Maternal depression and child psychopathology: A meta-analytic review. Clinical Child and Family Psychology Review, 14(1), 1-27.

Gross, H. E., Shaw, D. S., Burwell, R. A., \& Nagin, D. S. (2009). Transactional processes in child disruptive behavior and maternal depression: A longitudinal study from early childhood to adolescence. Development and Psychopathology, 21(1), 139-156.

Hammen, C., Bistricky, S. L., \& Ingran, R. (2010). Vulnerability to depression in adulthood. Em R. Ingram \& J. M. Price (Eds.), Vulnerability to psychopathology - Risks across the lifespan (pp. 248-281). New York: Guilford Press.

Jenkins, J. M., \& Curwen, T. (2008). Change in adolescents' internalizing symptomatology as a function of sex and the timing of maternal depressive symptomatology. Journal of the American Academy of Child and Adolescent Psychiatry, 47(4), 399-405.

Loosli, L. (2011). Depressão materna e o perfil de socialização de meninos e meninas em idade escolar (Dissertação de Mestrado). Programa de Pós-Graduação em Saúde Mental, Universidade de São Paulo, Ribeirão Preto, São Paulo, Brasil.

Loosli, L., \& Loureiro, S. R. (2010). Associação entre depressão materna e diferenças de gênero no comportamento de crianças: Uma revisão sistemática. Revista de Psiquiatria do Rio Grande do Sul, 32(3), 94-101.

Marturano, E. M., Toller, G. P., \& Elias, L. C. S. (2005). Gênero, adversidade e problemas socioemocionais associados à queixa escolar. Estudos de Psicologia, 22(4), 371-380. 
Mian, L., Tango. L. A., Lopes, J., \& Loureiro, S. R. (2009). A depressão materna e o comportamento de crianças em idade escolar. Psicologia: Teoria e Pesquisa, 25(1), 29-37.

Organização Mundial de Saúde. (2012). Depression (Fact sheet N³69). Recuperado de http://www.who.int/mediacentre/ factsheets/fs369/en/

Organização Mundial da Saúde. (2014). CID-10: Classificação Estatística Internacional de Doenças e Problemas Relacionados à Saúde-10 . revisão. São Paulo: Edusp. (Original publicado em 1989)

Papalia, D. E., \& Feldman, R. D. (2013). Desenvolvimento Humano (12 ed.). Porto Alegre: AMGH.

Shaw, D. S., Hyde, L. W., \& Brennan, L. M. (2012). Early predictors of boys' antisocial trajectories. Development and Psychopathology, 24(3), 871-888.

Silk, J. S., Shaw, D. S., Skuban, E. M., Oland, A. A., \& Kovacs, M. (2006). Emotion regulation strategies in offspring of childhoodonset depressed mothers. Journal of Child Psychology and Psychiatry, 47(1), 69-78.
Silverstein, M., Augustyn, M., Cabral, H., \& Zuckerman, B. (2006). Maternal depression and violence exposure: Double jeopardy for child school functioning. Pediatrics, 118(3), 792-800.

Stein, L. M. (1994). TDE - Teste de desempenho escolar: Manual para aplicação e interpretação. São Paulo: Casa do Psicólogo.

Tompson, M. C., Pierre, C. B., Boger, K. D., McKowen, J. W., Chan, P. T., \& Freed, R. D. (2010). Maternal Depression, maternal expressed emotion and youth psychopathology. Journal of Abnormal Child Psychology, 38(1), 105-117.

Toth, S. L., \& Cicchetti, D. (2010). The historical origins and developmental pathways of the discipline of developmental psychopathology. The Israel Journal of Psychiatry and Related Sciences, 47(2), 95-104.

Woerner, W., Fleitlich-Bilyk, B., Martinussen, R., Fletcher, J., Cucchiaro, G., Dalgalarrondo, P., .. . Tannock, R. (2004). The Strengths and Difficulties Questionnaire overseas: Evaluations and applications of the SDQ beyond Europe. European Child \& Adolescent Psychiatry, 13(2), II/47 - II/54.

Recebido em 18.04.2012

Primeira decisão editorial em 06.03.2015

Versão final em 04.02.2016

Aceito em 23.03.2016 